\title{
C-12-Substituted Indolo[2,1-a]isoquinolines as Estrogen Receptor Affinic Cytostatic Agents
}

\author{
Reinhard Ambros, Silvia von Angerer*, and \\ Wolfgang Wiegrebe
}

Institut für Pharmazie, Lehrstuhl Pharmazeutische Chemie I and Sonderforschungsbereich 234, Universität Regensburg, Universitätsstr. 31, D-8400 Regensburg, Germany

Received February 16, 1988

Methoxysubstituted 5,6-dihydro-indolo[2,1-a]isoquinolines with a methyl (2b-f) or a formyl group at C-12 (4a-f) and 12,12-dimethylisoquinolinium salts (3b-f) were synthesized and tested for cytostatic activity in vitro. The tetramethoxy-indoloisoquinoline $\mathbf{4 f}$ was the most active derivative in the $P$ $388 \mathrm{D}_{1}$ leukemia cell line, whereas compounds with two methoxy groups $(\mathbf{4 a}, \mathbf{4 b})$ were more potent against the MDA-MB 231 mammary tumor cells. The tetraacetoxy-12-formyl-5,6-dihydro-indoloisoquinoline 9 has proven to be active in both cell lines $(\mathrm{T} / \mathrm{C}=5 \%)$. In vivo it increased the life span of mice with $P 388$ leukemia (T/C =133\%). The acetates 7 and 8 exhibited binding affinities for the estrogen receptor, but did not exert a selective action on hormone-dependent MCF-7 cells.
In 12-Stellung substituierte Indolo[2,1-a]isochinoline als östrogenrezeptoraffine Cytostatika

Methoxysubstituierte 5,6-Dihydro-indolo[2,1-a]isochinoline mit einer Methyl- (2b-f) oder Formylgruppe an C-12 (4a-f), sowie die 12,12-Dimethylisochinoliniumsalze $\mathbf{3 b}-\mathbf{f}$ wurden synthetisiert und auf cytostatische Wirksamkeit in vitro getestet. Das Tetramethoxyindoloisochinolin $\mathbf{4 f}$ war die aktivste Substanz an P $388 \mathrm{D}_{1}$-Leukaemiezellen, während Verbindungen mit zwei Methoxygruppen (4a, 4b) an der menschlichen MDA-MB 231-Zellinie wirksamer waren. Das Tetraacetoxy-12-formyl-5,6-dihydroindoloisochinolin 9 war an beiden Zellinien aktiv $(\mathrm{T} / \mathrm{C}=5 \%)$. In vitro verlängerte 9 die Überlebenszeit von Mäusen mit P 388-Leukaemie (T/C $=133 \%$ ). Die Acetate 7 und 8 zeigten Affinität zum Östrogenrezeptor, aber bewirkten keine selektive Hemmung an hormonabhängigen MCF-7 Zellen.
In a previous paper we described the synthesis of a number of methoxysubstituted indolo[2,1-a]isoquinolines and their dihydro derivatives 1a-f $\mathbf{f}^{1}$ ). Their cytostatic activity was too low for the intended development of cytostatic agents binding to the estrogen receptor.

Therefore, we tried to increase their activity by introduction of an additional substituent in position 12 of the tetracycle. Two different substituents were considered: the methyl group in order to increase the lipophilicity and a formyl group to decrease the electron density at the nitrogen. A further step was the conversion of the indoloisoquinolines to quaternary salts. All of the compounds were tested for their cytostatic activity in vitro using MDA-MB 231 mammary tumor cells and P $388 \mathrm{D}_{1}$ leukemia cells. Since a prerequisite for the binding to the estrogen receptor is the presence of hydroxy or acetoxy groups in the aromatic rings, the methoxy groups of some derivatives were cleaved. After conversion to the acetates, their binding affinities for the estrogen receptor (ER) were determined.

\section{Chemistry}

The methoxy substituted 12-methyl-5,6-dihydro-indolo[ 2,1 -a $]$ isoquinolines $\mathbf{2} \mathbf{b}-\mathbf{f}$ were obtained by reacting $\mathbf{1 b}-\mathbf{f}^{\mathbf{1})}$ with an excess of $\mathrm{CH}_{3} \mathrm{I}$ at $90-93^{\circ}$ in a sealed tube. Increasing the temp. to $110^{\circ}$ led to the quaternary isoquinolinium salts 3b-f. The formation of quaternary $\mathrm{N}$-methyl compounds can be ruled out by ${ }^{1} \mathrm{H}-\mathrm{NMR}$ spectroscopy. The spectra showed only one singulett for the two methyl groups at C-12 at $\delta 1.85$. The formyl group in compound $\mathbf{4 a - f}$ was introduced in 1a-f by a Vilsmeier-Haack reaction using DMF/ $\mathrm{POCl}_{3}$. The regiospecific attack at position 12 was confirmed by spectroscopy. In the ${ }^{1} \mathrm{H}-\mathrm{NMR}$ spectra the vinyl proton has disappeared and the IR-spectra showed a carbonyl vibration at $1635 \mathrm{~cm}^{-1}$, a value characteristic for vinylogous amides $^{2}$. Compounds $2 \mathbf{f}$ and $\mathbf{4 f}$ were dehydrogenated to the aromatic indolo[2,1-a] isoquinolines 5 and 6 using Pd/C. For<smiles>[R]c1cc2cc3n(c2cc1[R])CCc1cc(OC)c([R])cc1-3</smiles><smiles>[R]c1cc2c(C)c3n(c2cc1[R])CCc1cc(OC)c([R])cc1-3</smiles><smiles>[R]c1cc2c(CO)c3n(c2cc1[R])CCc1cc(OC)c([R])cc1-3</smiles><smiles>[R]c1cc2c(cc1[R])[N+]([O-])=C1C(C)(C)c3cc(OC)c([R])cc3[N+]1([O-])CC2</smiles>

\begin{tabular}{r|lll} 
1-4 & $\mathrm{R}^{1}$ & $\mathrm{R}^{2}$ & $\mathrm{R}^{3}$ \\
\hline $\mathbf{a}$ & $\mathrm{H}$ & $\mathrm{H}$ & $\mathrm{OCH}_{3}$ \\
$\mathbf{b}$ & $\mathrm{H}$ & $\mathrm{OCH}_{3}$ & $\mathrm{H}$ \\
c & $\mathrm{H}$ & $\mathrm{OCH}_{3}$ & $\mathrm{OCH}_{3}$ \\
d & $\mathrm{OCH}_{3}$ & $\mathrm{H}$ & $\mathrm{OCH}_{3}$ \\
e & $\mathrm{OCH}_{3}$ & $\mathrm{OCH}_{3}$ & $\mathrm{H}$ \\
f & $\mathrm{OCH}_{3}$ & $\mathrm{OCH}_{3}$ & $\mathrm{OCH}_{3}$
\end{tabular}<smiles>[R]c1c2cc(OC)c(OC)cc2n2ccc3cc(OC)c(OC)cc3c12</smiles><smiles>[R]c1cc2c(cc1OC(C)=O)CCn1c-2c([R4])c2cc(OC)c(OC)cc21</smiles>

$$
5 \mathrm{R}=\mathrm{CH}_{3}
$$$$
6 \mathrm{R}=\mathrm{CHO}
$$

$7 \mathrm{R}^{1}=\mathrm{R}^{2}=\mathrm{H}, \mathrm{R}^{3}=\mathrm{CH}_{3}$

$8 R^{1}=R^{2}=H, R^{3}=C H O$

$9 \mathrm{R}^{1}=\mathrm{R}^{2}=\mathrm{OCOCH}_{3}, \mathrm{R}^{3}=\mathrm{CHO}$ 
the determination of the binding affinities of $\mathbf{2 b}, \mathbf{4 b}$ and $\mathbf{4 f}$ for the ER, the methoxy groups were cleaved by $\mathrm{BBr}_{3}$. The resulting phenols were converted to the acetates $\mathbf{7}, \mathbf{8}$, and $\mathbf{9}$, because of their better stability.

\section{Cytostatic Activity and Receptor Affinity}

Three cell lines were used for the determination of cytostatic activity: $P 388 D_{1}$ cells deriving from a mouse leukemia, hormone-independent MDA-MB 231 and hormone-dependent MCF-7 mammary tumor cells of human origin. All of the new indoloisoquinolines were tested for cytostatic effects at a concentration $10^{-5}$ molar. The inhibition of cell growth was measured by cell counting and ${ }^{3} \mathrm{H}$-thymidine labeling. Like the starting 5,6-dihydro-indolo[2,1-alisoquinolines 1a-f the 12-methyl derivatives $\mathbf{2 b}-\mathbf{f}$ showed no significant inhibitory effect in both cell lines. The aromatic compound $\mathbf{5}$ was also devoid of cytostatic activity. In the series of the dimethyl-5,6-dihydro-indoloisoquinolinium salts $\mathbf{3 b}-\mathbf{f}$, one compound (3b) was active against $\mathrm{P} 388 \mathrm{D}_{1}$ cells $(\mathrm{T} / \mathrm{C}=45 \%)$ (Table 1). The introduction of a formyl group into position 12 led to a marked increase in cytostatic activity both in $\mathrm{P}$

Tab. 1: Effect of $3 b-f$ on the Growth of MDA-MB 231 and P 388 $\mathrm{D}_{1}$ Cells

\begin{tabular}{|c|c|c|c|c|}
\hline Compound $^{\text {a) }}$ & $\begin{array}{l}{ }^{\mathrm{P}} \\
\text { Cell no. } \\
\% \mathrm{~T} / \mathrm{C}^{\mathrm{b})}\end{array}$ & $\begin{array}{l}388 \mathrm{D}_{1} \\
{ }^{3} \mathrm{H} \text {-thymidine } \\
\text { incorp. } \\
\left.\% \mathrm{~T} / \mathrm{C}^{\mathrm{b}}\right)\end{array}$ & $\begin{array}{l}\text { MDA } \\
\text { Cell no. } \\
\left.\% \mathrm{~T} / \mathrm{C}^{\mathrm{b}}\right)\end{array}$ & $\begin{array}{l}-\mathrm{MB} 231 \\
{ }^{3} \mathrm{H} \text {-thymidine } \\
\text { incorp. } \\
\left.\% \mathrm{~T} / \mathrm{C}^{\mathrm{b}}\right)\end{array}$ \\
\hline $3 b$ & 45 & 45 & 87 & 55 \\
\hline $3 b^{c)}$ & 85 & 85 & & \\
\hline $3 c$ & 85 & 75 & 90 & 92 \\
\hline $3 d$ & 85 & 83 & 90. & 91 \\
\hline $3 e$ & 73 & 73 & 90 & 90 \\
\hline $3 f$ & 75 & 67 & 99 & 97 \\
\hline
\end{tabular}

a) Concentration $10^{-5} \mathrm{M}$.

b) $\% \mathrm{~T} / \mathrm{C}=$ test compound/control, $\times 100$; mean of three tests with six dishes or test tubes.

c) Concentration $10^{-6} \mathrm{M}$.

Tab. 2: Effect of 12-Formyl-indoloisoquinolines $4 a-f, 6,8$ and 9 on the Growth of MDA-MB 231 and P $388 \mathrm{D}_{1}$ Cells

\begin{tabular}{|c|c|c|c|c|}
\hline Compound ${ }^{a)}$ & $\begin{array}{l}\text { P } \\
\text { Cell no. } \\
\left.\% \mathrm{~T} / \mathrm{C}^{\mathrm{b}}\right)\end{array}$ & $\begin{array}{l}88 \mathrm{D}_{1} \\
{ }^{3} \mathrm{H} \text {-thymidine } \\
\text { incorp. } \\
\left.\% \mathrm{~T} / \mathrm{C}^{\mathrm{b}}\right)\end{array}$ & $\begin{array}{l}\text { MDA } \\
\text { Cell no. } \\
\% \mathrm{~T} / \mathrm{C}^{\mathrm{b})}\end{array}$ & $\begin{array}{c}-\mathrm{MB} 231 \\
{ }^{3} \mathrm{H} \text {-thymidine } \\
\text { incorp. } \\
\% \mathrm{~T} / \mathrm{C}^{\mathrm{b})}\end{array}$ \\
\hline $4 a$ & 52 & 25 & 25 & 6 \\
\hline $4 b$ & 52 & 48 & 24 & 2 \\
\hline $4 c$ & 78 & 25 & 53 & 7 \\
\hline $4 d$ & 70 & 39 & 69 & 12 \\
\hline $4 e$ & 25 & 10 & 35 & 4 \\
\hline $4 f$ & 20 & 7 & 64 & 4 \\
\hline $4 f^{c)}$ & 80 & 50 & & \\
\hline 6 & 70 & 86 & 20 & 20 \\
\hline 8 & 88 & 82 & 40 & 2 \\
\hline 9 & 5 & 0 & 10 & 0 \\
\hline 9c) & 23 & 23 & 40 & 30 \\
\hline 9d) & 63 & 80 & & \\
\hline $9^{e)}$ & 82 & 91 & & \\
\hline
\end{tabular}

a) Concentration $10^{-5} \mathrm{M}$.

b) $\% \mathrm{~T} / \mathrm{C}=$ test compound/control, $\times 100$; mean of three tests with six dishes or test tubes.

c) Concentration $5 \times 10^{-6} \mathrm{M}$.

d) Concentration $10^{-6} \mathrm{M}$.

e) Concentration $5 \times 10^{-7} \mathrm{M}$.
$388 \mathrm{D}_{1}$ leukemia and MDA-MB 231 mammary tumor cells (Table 2). In all cases the inhibition of the ${ }^{3} \mathrm{H}$-thymidine incorporation exceeded the effect on cell number. The tetramethoxy-indoloisoquinoline $\mathbf{4 f}$ was the most active methoxy derivative against leukemia cells, whereas compounds with two methoxy groups $(\mathbf{4 a}, \mathbf{4 b})$ were more potent in mammary tumor cells. Differences between the two cell lines were also observed with 6 and 8.

Since the methoxy compounds generally do not bind to the estrogen receptor, we converted in a preliminary study three derivatives to the corresponding acetates 7-9. In order to prove that this conversion is not accompanied by a loss of cytostatic activity we tested compound 9 in both hormone-independent cell lines. Interestingly, the acetate 9 was more active than the corresponding methoxy derivative $\mathbf{4 f}$ (Table 2).

The binding affinities of 7-9 for the estrogen receptor were measured by a competitive binding assay with $\left[{ }^{3} \mathrm{H}\right] 17 \beta$ estradiol. Calf uterine cytosol was used as receptor source and the dextran coated-charcoal (DCC) method was applied. The relative binding affinities (RBA) are given as the ratio of the molar concentrations of $17 \beta$-estradiol and indoloisoquinoline required to decrease the receptor bound radioactivity by $50 \%$, multiplied by 100 . Only compounds $7(\mathrm{RBA}=0.2)$ and $8(\mathrm{RBA}=0.4)$ exhibited a moderate receptor affinity, whereas the tetraacetate 9 did not bind to the receptor. This observation is in accordance with results obtained with 2-phenylindoles ${ }^{3}$.

The two derivatives with binding affinity for the estrogen receptor were tested for specific action against estrogen receptor positive human MCF-7 mammary tumor cells. No improvement of cytostatic activity was found in comparison to hormon-independent cells. Compound 7 showed no inhibitory effect, whereas 8 inhibited the growth of MCF-7 cells at $10^{-5} \mathrm{M}$ by $60 \%$ (cell number) and $83 \%\left(\left[{ }^{3} \mathrm{H}\right]\right.$ thymidine incorporation), respectively. The strong cytostatic effect of 9 in vitro prompted us to determine the antitumor activity in vivo using the $P 388$ leukemia of the mouse. At a dose of $40 \mathrm{mg} / \mathrm{kg}$, the increase of life span was $33 \%$. This value is above the limit set by the NCI for cytostatic activity ${ }^{4}$.

Tab. 3: Effect of 9 on the Growth of P 388 Leukemia in vivo

\begin{tabular}{|c|c|c|c|c|c|}
\hline Compound & $\begin{array}{c}\left.\text { dose }^{a}\right) \\
(\mathrm{mg} / \mathrm{kg})\end{array}$ & $\begin{array}{l}\text { Change of } \\
\text { body weight } \mathrm{b}) \\
(\mathrm{T}-\mathrm{C}) ;(\mathrm{g})\end{array}$ & $\begin{array}{l}\text { Survival } \\
\text { median } \\
\text { (d) }\end{array}$ & $\begin{array}{l}1 \text { timec) } \\
\text { range } \\
\text { (d) }\end{array}$ & $\left.\% \mathrm{~T} / \mathrm{C}^{\mathrm{d}}\right)$ \\
\hline 9 & $\begin{array}{l}10 \\
20 \\
40\end{array}$ & $\begin{array}{r}-0.9 \\
-0.4 \\
0.0\end{array}$ & $\begin{array}{l}10 \\
11 \\
12\end{array}$ & $\begin{array}{r}9-12 \\
10-14 \\
11-13\end{array}$ & $\begin{array}{l}111 \\
122 \\
133\end{array}$ \\
\hline control & - & - & 9 & $9-10$ & - \\
\hline cis-DDPe) & 4 & 1.5 & 21 & $18-26$ & 213 \\
\hline
\end{tabular}

a-d) See exp. part.

e) cis-diammine-dichloro-platinum-(II).

\section{Discussion}

The structure activity studies of a number of substituted indolo[2,1-a $]$ isoquinolines revealed, that only the introduction of a formyl group into position 12 leads to derivatives with a strong cytostatic activity against $P 388 \mathrm{D}_{1}$ murine leukemia and MDA-MB 231 human mammary tumor cells. For derivative 9 this effect was confirmed in vivo using the $\mathbf{P} 388$ 
leukemia of the mouse. Since the aim of these studies is the development of cytostatic agents binding to the estrogen receptor three derivatives were converted into the acetates. Their binding affinities to the estrogen receptor were presumably too low to exert a selective action on estrogen sensitive human MCF-7 tumor cells.

The authors thank C. Braun and M. Beer for technical assistance and the Deutsche Forschungsgemeinschaft (SFB 234) for financial support.

\section{Experimental Part}

Melting points: Büchi 510 apparatus, uncorrected. - Elemental analyses: Mikroanalytisches Laboratorium, University of Regensburg. - IR-spectra: Beckman Acculab 3, KBr. $-{ }^{1} \mathrm{H}-\mathrm{NMR}$ spectra: Varian EM $360 \mathrm{~L}$, Varian EM 390 or Bruker WM $250, \mathrm{CDCl}_{3}$, TMS as internal standard, 60 $\mathrm{MHz}$, if not stated otherwise. UV-spectra: Uvikon 810 Kontron. - Mass spectra: Varian MAT CH5. - Column Chromatography (CC): Kieselgel 60 (Merck). - Temp. in ${ }^{\circ} \mathrm{C}$.

General Procedure for the Synthesis of 5,6-Dihydro-12-methyl-indolo[2,1-a/isoquinolines $\mathbf{2 b - \mathbf { f }}$

5,6-Dihydro-methoxy-indoloisoquinoline $(1.0 \mathrm{mmol})$ in $2.5 \mathrm{ml}$ absol. $\mathrm{MeOH}$ and $2.5 \mathrm{ml} \mathrm{CH}_{3} \mathrm{I}$ was heated in a sealed tube to $90-93^{\circ}$ for $3 \mathrm{~h}$. After cooling, the solvent was removed and the residue was purified by $\mathrm{CC}$ $\left(\mathrm{SiO}_{2} ; \mathrm{CH}_{2} \mathrm{Cl}_{2}\right)$. Recrystallization from EtOH yielded colorless crystals. The yields were between 50 and $60 \%$.

5,6-Dihydro-3,9-dimethoxy-12-methyl-indolo/2,1-alisoquinoline (2b)

M. p. $105-106^{\circ} .-\mathrm{C}_{19} \mathrm{H}_{19} \mathrm{NO}_{2}$ (293.3) Calc. C $77.8 \mathrm{H} 6.50$ Found C 77.3 H 6.56. $-{ }^{1} \mathrm{H}-\mathrm{NMR}: \delta(\mathrm{ppm})=2.57\left(\mathrm{~s} ; 3 \mathrm{H},-\mathrm{CH}_{3}\right), 3.09(\mathrm{t} ; \mathrm{J}=7 \mathrm{~Hz}, 2 \mathrm{H}$, $\left.-\mathrm{CH}_{2}-\right), 3.86\left(\mathrm{~s} ; 3 \mathrm{H},-\mathrm{OCH}_{3}\right), 3.9\left(\mathrm{~s} ; 3 \mathrm{H},-\mathrm{OCH}_{3}\right), 4.14(\mathrm{t} ; \mathrm{J}=7 \mathrm{~Hz}, 2 \mathrm{H}$, $\left.-\mathrm{CH}_{2}-\right), 6.67-7.0(\mathrm{~m} ; 4 \mathrm{H}, \mathrm{ArH}), 7.45(\mathrm{~d} ; \mathrm{J}=9 \mathrm{~Hz}, 1 \mathrm{H}, \mathrm{ArH}), 7.75(\mathrm{~d} ; \mathrm{J}=$ $9 \mathrm{~Hz}, 1 \mathrm{H}, \mathrm{ArH}) .-\mathrm{MS}: \mathrm{m} / \mathrm{z}=293\left(100 \%, \mathrm{M}^{+\cdot}\right), 278\left(84 \%,{ }^{*} 263.76\right)$. $\mathrm{UV}\left(\mathrm{CH}_{3} \mathrm{CN}\right): \lambda \max (\log \varepsilon)=346(4.39), 331(4.45), 263(4.26) \mathrm{nm}$.

5,6-Dihydro-3,9,10-trimethoxy-12-methyl-indolo/2,1-a/isoquinoline (2c)

M. p. $174^{\circ} .-\mathrm{C}_{20} \mathrm{H}_{21} \mathrm{NO}_{3}$ (323.4) Calc. C $74.3 \mathrm{H} 6.55$ Found C 74.7 H 6.70. $-{ }^{1} \mathrm{H}-\mathrm{NMR}(90 \mathrm{MHz}): \delta(\mathrm{ppm})=2.55\left(\mathrm{~s} ; 3 \mathrm{H},-\mathrm{CH}_{3}\right), 3.08(\mathrm{t} ; \mathrm{J}=$ $\left.7 \mathrm{~Hz}, 2 \mathrm{H},-\mathrm{CH}_{2}-\right), 3.85\left(\mathrm{~s} ; 3 \mathrm{H},-\mathrm{OCH}_{3}\right), 3.97\left(\mathrm{~s} ; 6 \mathrm{H},-\mathrm{OCH}_{3}\right), 4.13(\mathrm{t}$; $\left.\mathrm{J}=7 \mathrm{~Hz}, 2 \mathrm{H},-\mathrm{CH}_{2}-\right), 6.79(\mathrm{~s} ; 1 \mathrm{H}, \mathrm{ArH}), 6.84(\mathrm{~s} ; 1 \mathrm{H}, \mathrm{ArH}), 6.90,7.74$ $(\mathrm{AB} ; \mathrm{J}=9 \mathrm{~Hz}, 2 \mathrm{H}, \mathrm{ArH}), 7.03(\mathrm{~s} ; 1 \mathrm{H}, \mathrm{ArH})$.

5,6-Dihydro-2,3,10-trimethoxy-12-methyl-indolo/2,1-alisoquinoline (2d)

M. p. $142^{\circ}$. $-\mathrm{C}_{20} \mathrm{H}_{21} \mathrm{NO}_{3}$ (323.4) Calc. C $74.3 \mathrm{H} 6.55$ Found $\mathrm{C} 74.1$ H 6.35. - ${ }^{1} \mathrm{H}-\mathrm{NMR}(90 \mathrm{MHz}): \delta(\mathrm{ppm})=2.6\left(\mathrm{~s} ; 3 \mathrm{H},-\mathrm{CH}_{3}\right), 3.05(\mathrm{t} ; \mathrm{J}=7$ $\left.\mathrm{Hz}, 2 \mathrm{H},-\mathrm{CH}_{2}-\right), 3.86\left(\mathrm{~s} ; 3 \mathrm{H},-\mathrm{OCH}_{3}\right), 3.92\left(\mathrm{~s} ; 3 \mathrm{H},-\mathrm{OCH}_{3}\right), 3.96(\mathrm{~s} ; 3 \mathrm{H}$, $\left.-\mathrm{OCH}_{3}\right), 4.15\left(\mathrm{t} ; \mathrm{J}=7 \mathrm{~Hz}, 2 \mathrm{H},-\mathrm{CH}_{2}-\right), 6.73-7.1(\mathrm{~m} ; 4 \mathrm{H}, \mathrm{ArH}), 7.34(\mathrm{~s}$; 1H, ArH).

\section{5,6-Dihydro-2,3,9-trimethoxy-12-methyl-indolo/2,1-a/isoquinoline (2e)}

M. p. $150^{\circ}$. $-\mathrm{C}_{20} \mathrm{H}_{21} \mathrm{NO}_{3}$ (323.4) Calc. C $74.3 \mathrm{H} 6.55$ Found $\mathrm{C} 74.5$ H 6.40. $-{ }^{1} \mathrm{H}-\mathrm{NMR}: \delta(\mathrm{ppm})=2.59 .\left(\mathrm{s} ; 3 \mathrm{H},-\mathrm{CH}_{3}\right), 3.05(\mathrm{t} ; \mathrm{J}=7 \mathrm{~Hz}, 2 \mathrm{H}$, $\left.-\mathrm{CH}_{2}-\right), 3.88\left(\mathrm{~s} ; 3 \mathrm{H},-\mathrm{OCH}_{3}\right), 3.93\left(\mathrm{~s} ; 3 \mathrm{H},-\mathrm{OCH}_{3}\right), 3.99(\mathrm{~s} ; 3 \mathrm{H}$, $\left.-\mathrm{OCH}_{3}\right), 4.16\left(\mathrm{t} ; \mathrm{J}=7 \mathrm{~Hz}, 2 \mathrm{H},-\mathrm{CH}_{2}-\right), 6.76-6.86(\mathrm{~m} ; 3 \mathrm{H}, \mathrm{ArH}), 7.40(\mathrm{~s}$; $1 \mathrm{H}, \operatorname{ArH}), 7.47$ (d; J $=9 \mathrm{~Hz}, 1 \mathrm{H}, \operatorname{ArH})$.

5,6-Dihydro-2,3,9,10-tetramethoxy-12-methyl-indolo/ 2,1-a/isoquinoline (2f)

M. p. 216-217.$-\mathrm{C}_{21} \mathrm{H}_{23} \mathrm{NO}_{4}(353.4)$ Calc. C $71.4 \mathrm{H} 6.55$ Found C 71.0 H 6.72. $-{ }^{1} \mathrm{H}-\mathrm{NMR}(90 \mathrm{MHz}): \delta(\mathrm{ppm})=2.56\left(\mathrm{~s} ; 3 \mathrm{H},-\mathrm{CH}_{3}\right), 3.05(\mathrm{t} ; \mathrm{J}=$ $\left.7 \mathrm{~Hz}, 2 \mathrm{H},-\mathrm{CH}_{2}-\right), 3.92\left(\mathrm{~s} ; 12 \mathrm{H},-\mathrm{OCH}_{3}\right), 4.07\left(\mathrm{t} ; \mathrm{J}=7 \mathrm{~Hz}, 2 \mathrm{H},-\mathrm{CH}_{2}-\right)$, $6.7(\mathrm{~s} ; 2 \mathrm{H}, \operatorname{ArH}), 6.93(\mathrm{~s} ; 1 \mathrm{H}, \operatorname{ArH}), 7.27$ (s;1H, ArH).

General Procedure for the Synthesis of 5,6-Dihydro-12,12-dimethylindolo/ 2,1-a/isoquinolinium Iodides $\mathbf{3 b - f}$

The isoquinolinium salts were prepared in the same way as described for the monomethyl compounds, only the reaction temp. was raised to $110^{\circ}$ for $4 \mathrm{~h}$. After work-up the precipitate was washed with ether: yellow crystals. The yields ranged from 65 to $75 \%$.

\section{5,6-Dihydro-3,9-dimethoxy-12,12-dimethyl-indolo/2,1-alisoquinolinium} iodide (3b)

M. p. $220^{\circ}$ dec. $-\mathrm{C}_{20} \mathrm{H}_{22} \mathrm{NO}_{2} \cdot \mathrm{I} \times \mathrm{H}_{2} \mathrm{O}(453.3)$ Valc. C $53.1 \mathrm{H} 4.86$ Found C 53.0 H 4.89. - IR (KBr): $3450\left(\mathrm{H}_{2} \mathrm{O}\right), 1630(\mathrm{C}=\mathrm{N}) \mathrm{cm}^{-1} \cdot{ }^{1}{ }^{1} \mathrm{H}-$ NMR $(90 \mathrm{MHz}): \delta(\mathrm{ppm})=1.87\left(\mathrm{~s} ; 6 \mathrm{H},-\mathrm{CH}_{3}\right), 3.64(\mathrm{t} ; \mathrm{J}=7 \mathrm{~Hz}, 2 \mathrm{H}$, $\left.-\mathrm{CH}_{2}-\right), 4.0\left(\mathrm{~s} ; 3 \mathrm{H},-\mathrm{OCH}_{3}\right), 4.04\left(\mathrm{~s} ; 3 \mathrm{H},-\mathrm{OCH}_{3}\right), 4.97(\mathrm{t} ; \mathrm{J}=7 \mathrm{~Hz}, 2 \mathrm{H}$, $\left.-\mathrm{CH}_{2}-\right), 7.03-7.23(\mathrm{~m} ; 3 \mathrm{H}, \mathrm{ArH}), 7.47(\mathrm{~d} ; \mathrm{J}=9 \mathrm{~Hz}, 1 \mathrm{H}, \mathrm{ArH}), 7.54(\mathrm{~s}$; $1 \mathrm{H}, \operatorname{ArH}), 8.16(\mathrm{~d} ; \mathrm{J}=9 \mathrm{~Hz}, 1 \mathrm{H}, \operatorname{ArH})$.

5,6-Dihydro-3,9,10-trimethoxy-12,12-dimethyl-indolo/2,1-alisoquinolinium iodide (3c)

M. p. $226^{\circ}$ dec. $-\mathrm{C}_{21} \mathrm{H}_{24} \mathrm{NO}_{3} \cdot \mathrm{I} \times \mathrm{H}_{2} \mathrm{O}(483.3)$ Calc. C $52.2 \mathrm{H} 5.00$ Found C $51.7 \mathrm{H} 5.08 .-{ }^{1} \mathrm{H}-\mathrm{NMR}: \delta(\mathrm{ppm})=1.88\left(\mathrm{~s} ; 6 \mathrm{H},-\mathrm{CH}_{3}\right), 3.58(\mathrm{t}$; $\left.\mathrm{J}=7 \mathrm{~Hz}, 2 \mathrm{H},-\mathrm{CH}_{2}-\right), 4.0\left(\mathrm{~s} ; 6 \mathrm{H},-\mathrm{OCH}_{3}\right), 4.13\left(\mathrm{~s} ; 3 \mathrm{H},-\mathrm{OCH}_{3}\right), 5.0(\mathrm{t}$; $\left.\mathrm{J}=7 \mathrm{~Hz}, 2 \mathrm{H},-\mathrm{CH}_{2}-\right), 7.0-7.17(\mathrm{~m} ; 3 \mathrm{H}, \mathrm{ArH}), 7.67(\mathrm{~s} ; 1 \mathrm{H}, \mathrm{ArH}), 8.1(\mathrm{~d}$; $\mathrm{J}=9 \mathrm{~Hz}, 1 \mathrm{H}, \mathrm{ArH}) .-\mathrm{UV}(\mathrm{MeOH}): \lambda \max (\log \varepsilon)=434(4.19), 308$ (3.75), 247 (4.33) $\mathrm{nm}$.

5,6-Dihydro-2,3,10-trimethoxy-12,12-dimethyl-indolo/2,1-a/isoquinolinium iodide (3d)

M. p. $240^{\circ}$ dec. $-\mathrm{C}_{21} \mathrm{H}_{24} \mathrm{NO}_{3} \cdot \mathrm{I} \times \mathrm{H}_{2} \mathrm{O}$ (483.3) Calc. C $52.2 \mathrm{H} 5.00$ Found C $51.5 \mathrm{H} 4.94 .-{ }^{1} \mathrm{H}-\mathrm{NMR}: \delta(\mathrm{ppm})=1.93\left(\mathrm{~s} ; 6 \mathrm{H},-\mathrm{CH}_{3}\right), 3.66(\mathrm{t}$; $\left.\mathrm{J}=7 \mathrm{~Hz}, 2 \mathrm{H},-\mathrm{CH}_{2}-\right), 3.93\left(\mathrm{~s} ; 3 \mathrm{H},-\mathrm{OCH}_{3}\right), 4.0\left(\mathrm{~s} ; 3 \mathrm{H},-\mathrm{OCH}_{3}\right), 4.13(\mathrm{~s}$; $\left.3 \mathrm{H},-\mathrm{OCH}_{3}\right), 4.91\left(\mathrm{t} ; \mathrm{J}=7 \mathrm{~Hz}, 2 \mathrm{H},-\mathrm{CH}_{2}-\right), 7.03-7.27(\mathrm{~m} ; 3 \mathrm{H}, \mathrm{ArH})$, 7.43 (s; 1H, ArH), 7.87 (d; J = 9 Hz, 1H, ArH).

5,6-Dihydro-2,3,9-trimethoxy-12,12-dimethyl-indolo/2,1-a/isoquinolinium iodide (3e)

M. p. $210-211^{\circ}$ dec. $-\mathrm{C}_{21} \mathrm{H}_{24} \mathrm{NO}_{3} \cdot \mathrm{I} \times \mathrm{H}_{2} \mathrm{O}$ (483.3) Calc. C $52.2 \mathrm{H} 5.00$ Found C $51.9 \mathrm{H}$ 5.16. $-{ }^{1} \mathrm{H}-\mathrm{NMR}: \delta(\mathrm{ppm})=1.85\left(\mathrm{~s} ; 6 \mathrm{H},-\mathrm{CH}_{3}\right), 3.63(\mathrm{t}$; $\left.\mathrm{J}=7 \mathrm{~Hz}, 2 \mathrm{H},-\mathrm{CH}_{2}-\right), 3.96\left(\mathrm{~s} ; 6 \mathrm{H},-\mathrm{OCH}_{3}\right), 4.08\left(\mathrm{~s} ; 3 \mathrm{H},-\mathrm{OCH}_{3}\right), 4.97$ $\left(\mathrm{t} ; \mathrm{J}=7 \mathrm{~Hz}, 2 \mathrm{H},-\mathrm{CH}_{2}-\right), 7.0\left(\mathrm{dd} ; \mathrm{J}_{1 / 2}=8 / 2 \mathrm{~Hz}, 1 \mathrm{H}, \mathrm{ArH}\right), 7.23(\mathrm{~s} ; 1 \mathrm{H}$, $\operatorname{ArH}), 7.3(\mathrm{~d} ; \mathrm{J}=8 \mathrm{~Hz}, 1 \mathrm{H}, \operatorname{ArH}), 7.36(\mathrm{~s} ; 1 \mathrm{H}, \operatorname{ArH}), 7.47(\mathrm{~d} ; \mathrm{J}=2 \mathrm{~Hz}$, $1 \mathrm{H}, \operatorname{ArH})$.

5,6-Dihydro-2,3,9,10-tetramethoxy-12,12-dimethyl-indolo/2,1-alisoquinolinium iodide (3f)

M. p. $232^{\circ}$ dec. $-\mathrm{C}_{22} \mathrm{H}_{26} \mathrm{NO}_{4} \cdot \mathrm{I} \times \mathrm{H}_{2} \mathrm{O}$ (512.3) Calc. C $51.5 \mathrm{H} 5.10$ Found C $51.0 \mathrm{H} 5.51 .-{ }^{1} \mathrm{H}-\mathrm{NMR}: \delta(\mathrm{ppm})=1.89\left(\mathrm{~s} ; 6 \mathrm{H},-\mathrm{CH}_{3}\right), 3.59(\mathrm{t}$; $\left.\mathrm{J}=7 \mathrm{~Hz}, 2 \mathrm{H},-\mathrm{CH}_{2}-\right), 4.0\left(\mathrm{~s} ; 6 \mathrm{H},-\mathrm{OCH}_{3}\right), 4.09\left(\mathrm{~s} ; 3 \mathrm{H},-\mathrm{OCH}_{3}\right), 4.12(\mathrm{~s}$; $\left.3 \mathrm{H},-\mathrm{OCH}_{3}\right), 5.03\left(\mathrm{t} ; \mathrm{J}=7 \mathrm{~Hz}, 2 \mathrm{H},-\mathrm{CH}_{2}-\right), 7.09(\mathrm{~s} ; 1 \mathrm{H}, \mathrm{ArH}), 7.17(\mathrm{~s}$; $1 \mathrm{H}, \mathrm{ArH}), 7.4$ (s; 1H, ArH), 7.67 (s; 1H, ArH).

General Procedure for the Synthesis of 12-Formyl-5,6-dihydro-indolo[2,1-a]isoquinolines $\mathbf{4 a - f}$

Absol. DMF $(0.6 \mathrm{ml})$ was added slowly to $\mathrm{POCl}_{3}(0.9 \mathrm{ml})$ at $10-20^{\circ}$ under $\mathrm{N}_{2}$. The mixture was stirred for $5 \mathrm{~min}$ at $15-20^{\circ}$. The 5,6-dihydro-metho$\mathrm{xy}$-indolol 2,1 -alisoquinoline $(1.0 \mathrm{mmol})$ dissolved in $5 \mathrm{ml}$ absol. DMF was added slowly to keep the temp. below $35^{\circ}$. After stirring for $35 \mathrm{~min}$ at $35^{\circ}$, the mixture was poured into $30 \mathrm{ml}$ ice water. The aqueous layer was basified $(\mathrm{NaOH})$, and extracted with $\mathrm{CHCl}_{3}$. After washing with water and drying $\left(\mathrm{Na}_{2} \mathrm{SO}_{4}\right)$, the solvent was removed in vacuo. The products 
were recrystallized from $\mathrm{MeOH}$ to give slightly yellow crystals. The yields were $70-90 \%$.

\section{2-Formyl-5,6-dihydro-3,10-dimethoxy-indolo/ 2,1-a/isoquinoline (4a)}

M. p. $160.5^{\circ}$. $-\mathrm{C}_{19} \mathrm{H}_{17} \mathrm{NO}_{3}$ (307.3) Calc. C $74.3 \mathrm{H} 5.57$ Found C 74.2 H 5.69. - IR (KBr): $1640(\mathrm{CO}) \mathrm{cm}^{-1}$. - ${ }^{1} \mathrm{H}-\mathrm{NMR}(90 \mathrm{MHz}): \delta(\mathrm{ppm})=$ $3.13\left(\mathrm{t} ; \mathrm{J}=7 \mathrm{~Hz}, 2 \mathrm{H},-\mathrm{CH}_{2}-\right), 3.88\left(\mathrm{~s} ; 3 \mathrm{H},-\mathrm{OCH}_{3}\right), 3.93(\mathrm{~s} ; 3 \mathrm{H}$, $\left.-\mathrm{OCH}_{3}\right), 4.2\left(\mathrm{t} ; \mathrm{J}=7 \mathrm{~Hz}, 2 \mathrm{H},-\mathrm{CH}_{2}-\right), 6.91(\mathrm{~s} ; 1 \mathrm{H}, \mathrm{ArH}), 6.95,7.27(\mathrm{AB}$; $\mathrm{J}=9 \mathrm{~Hz}, 2 \mathrm{H}, \operatorname{ArH}), 6.96(\mathrm{~d} ; \mathrm{J}=9 \mathrm{~Hz}, 1 \mathrm{H}, \operatorname{ArH}), 7.85(\mathrm{~d} ; \mathrm{J}=2 \mathrm{~Hz}, 1 \mathrm{H}$, ArH), 7.9 (dd; $\left.J_{1 / 2}=9 / 2 \mathrm{~Hz}, 1 \mathrm{H}, \mathrm{ArH}\right), 10.5$ (s; 1H, -CHO). - UV $\left(\mathrm{CH}_{3} \mathrm{CN}\right): \lambda \max (\log \varepsilon)=350(4.36), 272(4.47) \mathrm{nm}$.

\section{2-Formyl-5,6-dihydro-3,9-dimethoxy-indolo/ 2,1-a/isoquinoline (4b)}

M. p. $170.5^{\circ} .-\mathrm{C}_{19} \mathrm{H}_{17} \mathrm{NO}_{3}$ (307.3) Calc. C $74.3 \mathrm{H} 5.57$ Found $\mathrm{C} 74.3$ $\mathrm{H}$ 5.59. - ${ }^{1} \mathrm{H}-\mathrm{NMR}(90 \mathrm{MHz}): \delta(\mathrm{ppm})=3.1\left(\mathrm{t} ; \mathrm{J}=7 \mathrm{~Hz}, 2 \mathrm{H},-\mathrm{CH}_{2^{-}}\right)$, $3.87\left(\mathrm{~s} ; 6 \mathrm{H},-\mathrm{OCH}_{3}\right), 4.12\left(\mathrm{t} ; \mathrm{J}=7 \mathrm{~Hz}, 2 \mathrm{H},-\mathrm{CH}_{2}-\right), 6.73-7.0(\mathrm{~m} ; 4 \mathrm{H}$, $\operatorname{ArH}), 7.83(\mathrm{~d} ; \mathrm{J}=9 \mathrm{~Hz}, 1 \mathrm{H}, \mathrm{ArH}), 8.23(\mathrm{~d} ; \mathrm{J}=9 \mathrm{~Hz}, 1 \mathrm{H}, \mathrm{ArH}), 10.42(\mathrm{~s}$; $1 \mathrm{H},-\mathrm{CHO})$.

\section{2-Formyl-5,6-dihydro-3,9,10-trimethoxy-indolo/2,1-a/isoquinoline (4c)}

M. p. $161.5^{\circ} .-\mathrm{C}_{20} \mathrm{H}_{19} \mathrm{NO}_{4}$ (337.3) Calc. C $71.2 \mathrm{H} 5.67$ Found $\mathrm{C} 71.3$ H 5.56. $-{ }^{1} \mathrm{H}-\mathrm{NMR}: \delta(\mathrm{ppm})=3.12\left(\mathrm{t} ; \mathrm{J}=7 \mathrm{~Hz}, 2 \mathrm{H},-\mathrm{CH}_{2}-\right), 3.88(\mathrm{~s} ; 3 \mathrm{H}$, $\left.-\mathrm{OCH}_{3}\right), 3.97\left(\mathrm{~s} ; 3 \mathrm{H},-\mathrm{OCH}_{3}\right), 4.0\left(\mathrm{~s} ; 3 \mathrm{H},-\mathrm{OCH}_{3}\right), 4.14(\mathrm{t} ; \mathrm{J}=7 \mathrm{~Hz}, 2 \mathrm{H}$, $\left.-\mathrm{CH}_{2}-\right), 6.76-6.99(\mathrm{~m} ; 3 \mathrm{H}, \mathrm{ArH}), 7.70-7.95(\mathrm{~m} ; 2 \mathrm{H}, \mathrm{ArH}), 10.4(\mathrm{~s} ; 1 \mathrm{H}$, $-\mathrm{CHO})$.

\section{2-Formyl-5,6-dihydro-2,3,10-trimethoxy-indolo/2,1-a/isoquinoline (4d)}

M. p. $212^{\circ}$. $-\mathrm{C}_{20} \mathrm{H}_{19} \mathrm{NO}_{4}(337.3)$ Calc. C $71.2 \mathrm{H} 5.67$ Found $\mathrm{C} 71.4$ H 5.68. - ${ }^{1} \mathrm{H}-\mathrm{NMR}(90 \mathrm{MHz}): \delta(\mathrm{ppm})=3.09\left(\mathrm{t} ; \mathrm{J}=7 \mathrm{~Hz}, 2 \mathrm{H},-\mathrm{CH}_{2}-\right)$, $3.90\left(\mathrm{~s} ; 3 \mathrm{H},-\mathrm{OCH}_{3}\right), 3.94\left(\mathrm{~s} ; 3 \mathrm{H},-\mathrm{OCH}_{3}\right), 3.97\left(\mathrm{~s} ; 3 \mathrm{H},-\mathrm{OCH}_{3}\right), 4.16(\mathrm{t}$; $\left.\mathrm{J}=7 \mathrm{~Hz}, 2 \mathrm{H},-\mathrm{CH}_{2}-\right), 6.8(\mathrm{~s} ; 1 \mathrm{H}, \mathrm{ArH}), 6.87\left(\mathrm{dd} ; \mathrm{J}_{1 / 2}=9 / 2 \mathrm{~Hz}, 1 \mathrm{H}\right.$, $\operatorname{ArH}), 7.20(\mathrm{~d} ; \mathrm{J}=9 \mathrm{~Hz}, 1 \mathrm{H}, \operatorname{ArH}), 7.53(\mathrm{~s} ; 1 \mathrm{H}, \operatorname{ArH}), 7.84(\mathrm{~d} ; \mathrm{J}=2 \mathrm{~Hz}$, $1 \mathrm{H}, \mathrm{ArH}), 10.47$ (s; 1H, -CHO).

\section{2-Formyl-5,6-dihydro-2,3,9-trimethoxy-indolo/2,1-alisoquinoline (4e)}

M. p. $208^{\circ}$. $-\mathrm{C}_{20} \mathrm{H}_{19} \mathrm{NO}_{4}$ (337.3) Calc. C $71.2 \mathrm{H} 5.67$ Found $\mathrm{C} 70.8$ H 5.76. - ${ }^{1} \mathrm{H}-\mathrm{NMR}(90 \mathrm{MHz}): \delta(\mathrm{ppm})=3.09\left(\mathrm{t} ; \mathrm{J}=7 \mathrm{~Hz}, 2 \mathrm{H},-\mathrm{CH}_{2}-\right)$, $3.87\left(\mathrm{~s} ; 3 \mathrm{H},-\mathrm{OCH}_{3}\right), 3.97\left(\mathrm{~s} ; 6 \mathrm{H},-\mathrm{OCH}_{3}\right), 4.14(\mathrm{t} ; \mathrm{J}=7 \mathrm{~Hz}, 2 \mathrm{H}$, $\left.-\mathrm{CH}_{2}-\right), 6.84-6.9(\mathrm{~m} ; 2 \mathrm{H}, \mathrm{ArH}), 6.93\left(\mathrm{dd} ; \mathrm{J}_{1 / 2}=9 / 2 \mathrm{~Hz}, 1 \mathrm{H}, \mathrm{ArH}\right), 7.58$ (s; $1 \mathrm{H}, \mathrm{ArH}), 8.23(\mathrm{~d} ; \mathrm{J}=9 \mathrm{~Hz}, 1 \mathrm{H}, \mathrm{ArH}), 10.51$ (s; 1H, -CHO).

12-Formyl-5,6-dihydro-2,3,9,10-tetramethoxy-indolo/2,1-a/isoquinoline (4f)

M. p. $217.5^{\circ} .-\mathrm{C}_{21} \mathrm{H}_{21} \mathrm{NO}_{5}$ (367.4) Calc. C $68.7 \mathrm{H} 5.72$ Found $\mathrm{C} 68.3$ H 5.85. - ${ }^{1} \mathrm{H}-\mathrm{NMR}: \delta(\mathrm{ppm})=3.1\left(\mathrm{t} ; \mathrm{J}=7 \mathrm{~Hz}, 2 \mathrm{H},-\mathrm{CH}_{2}-\right), 3.98(\mathrm{~s} ; 12 \mathrm{H}$, $\left.-\mathrm{OCH}_{3}\right), 4.17\left(\mathrm{t} ; \mathrm{J}=7 \mathrm{~Hz}, 2 \mathrm{H},-\mathrm{CH}_{2}-\right), 6.8(\mathrm{~s} ; 1 \mathrm{H}, \mathrm{ArH}), 6.83(\mathrm{~s} ; 1 \mathrm{H}$, ArH), 7.5 (s; 1H, ArH), 7.9 (s; 1H, ArH), $10.5(\mathrm{~s} ; 1 \mathrm{H},-\mathrm{CHO})$.

\section{2,3,9,10-Tetramethoxy-12-methyl-indolo/2,1-a/isoquinoline (5)}

$2 \mathrm{f}(1.3 \mathrm{mmol})$ and $\mathrm{Pd} / \mathrm{C} 10 \%(150 \mathrm{mg})$ were mixed thoroughly in an agate mortar. This and all of the following operations were carried out under $\mathrm{N}_{2}$. A flask containing the mixture was placed in an oil bath of a temp. which was kept $10-15^{\circ}$ above the melting point of the dihydro compound. After $30 \mathrm{~min}$, the mixture was stirred with a spatula. Heating was continued for $30 \mathrm{~min}$. After cooling, the mixture was dissolved in $\mathrm{CH}_{2} \mathrm{Cl}_{2}$ and filtered. The solvent was evaporated. Recrystallization from $\mathrm{EtOH}$ afforded yellow crystals. - Yield $15 \%$; m. p. $230^{\circ}$ dec. $-\mathrm{C}_{21} \mathrm{H}_{21} \mathrm{NO}_{4} \times 1 / 4 \mathrm{H}_{2} \mathrm{O}$ (355.4) Calc. C 71.8 H 6.02 Found C 71.8 H 6.03. - IR (KBr): 3420 $\left(\mathrm{H}_{2} \mathrm{O}\right) \mathrm{cm}^{-1}$. - ${ }^{1} \mathrm{H}-\mathrm{NMR}: \delta(\mathrm{ppm})=2.76\left(\mathrm{~s} ; 3 \mathrm{H},-\mathrm{CH}_{3}\right), 3.93(\mathrm{~s} ; 3 \mathrm{H}$, $\left.-\mathrm{OCH}_{3}\right), 3.96\left(\mathrm{~s} ; 3 \mathrm{H},-\mathrm{OCH}_{3}\right), 4.01\left(\mathrm{~s} ; 3 \mathrm{H},-\mathrm{OCH}_{3}\right), 4.03(\mathrm{~s} ; 3 \mathrm{H}$, $\left.-\mathrm{OCH}_{3}\right), 6.50,7.83(\mathrm{AB} ; \mathrm{J}=9 \mathrm{~Hz}, 2 \mathrm{H}, \mathrm{ArH}), 6.82(\mathrm{~s} ; 1 \mathrm{H}, \mathrm{ArH}), 6.97$ (s; $1 \mathrm{H}, \mathrm{ArH}), 7.2$ (s; 1H, ArH), 7.8 (s; 1H, ArH). - MS: m/z = 351 (100\%,
$\left.\mathrm{M}^{+\cdot}\right), 336(60 \%, * 321.64), 175.5\left(15 \%, \mathrm{M}^{2+} / 2\right)$. - UV $\left(\mathrm{CH}_{3} \mathrm{CN}\right): \lambda \max$ $(\log \varepsilon)=372$ (3.99), 353 (4.05), 319 (4.49), 306 (4.34), 280 (4.57) nm.

\section{2-Formyl-2,3,9,10-tetramethoxy-indolo/2,1-a/isoquinoline (6)}

$\mathbf{6}$ was synthesized from $\mathbf{4 f}$ according to the procedure described above. Recrystallization from $\mathrm{MeOH}$ afforded yellow crystals. - Yield $50 \%$; m. p. $281^{\circ}$. $-\mathrm{C}_{21} \mathrm{H}_{19} \mathrm{NO}_{5} \times 1 / 2 \mathrm{H}_{2} \mathrm{O}(374.4)$ Calc. C $67.4 \mathrm{H} 5.38$ Found C 67.4 H 5.49. - IR (KBr): $3420\left(\mathrm{H}_{2} \mathrm{O}\right), 1630(\mathrm{CO}) \mathrm{cm}^{-1}$. - ${ }^{1} \mathrm{H}-\mathrm{NMR}$ $(250 \mathrm{MHz}): \delta(\mathrm{ppm})=4.03\left(\mathrm{~s} ; 3 \mathrm{H},-\mathrm{OCH}_{3}\right), 4.04\left(\mathrm{~s} ; 3 \mathrm{H},-\mathrm{OCH}_{3}\right), 4.06$ $\left(\mathrm{s} ; 3 \mathrm{H},-\mathrm{OCH}_{3}\right), 4.14\left(\mathrm{~s} ; 3 \mathrm{H},-\mathrm{OCH}_{3}\right), 7.04,8.05(\mathrm{AB} ; \mathrm{J}=9 \mathrm{~Hz}, 2 \mathrm{H}$, $\operatorname{ArH}), 7.07(\mathrm{~s} ; 1 \mathrm{H}, \mathrm{ArH}), 7.20(\mathrm{~s} ; 1 \mathrm{H}, \operatorname{ArH}), 7.27(\mathrm{~s} ; 1 \mathrm{H}, \operatorname{ArH}), 7.87(\mathrm{~s}$ $1 \mathrm{H}, \mathrm{ArH}), 10.68$ (s; 1H, -CHO). - MS: $\mathrm{m} / \mathrm{z}=365\left(100 \%, \mathrm{M}^{+} \cdot\right), 350$ (79 \%, *335.61), $335(5 \%), 182.5\left(14 \%, \mathrm{M}^{2+} / 2\right)$. - UV $\left(\mathrm{CH}_{3} \mathrm{CN}\right): \lambda \max$ $(\log \varepsilon)=409$ (4.32) 387 (4.21), 366 (3.97), 293 (4.35), 259 (4.50) nm.

\section{General Procedure for the Ether Cleavage and Acetylation}

$0.3 \mathrm{mmol}$ of the methoxysubstituted indoloisoquinoline in $5 \mathrm{ml}$ absol. $\mathrm{CH}_{2} \mathrm{Cl}_{2}$ was cooled to $-15^{\circ}$ under $\mathrm{N}_{2}$ and $\mathrm{BBr}_{3}(0.1 \mathrm{ml})$ was added. After stirring for $30 \mathrm{~min}$. the cooling bath was removed and the mixture was stirred for $15 \mathrm{~h}$. With cooling, the mixture was poured into $10 \mathrm{ml}$ of an aqueous solution of $\mathrm{NaHCO}_{3}$. Then $25 \mathrm{ml}$ EtOAc were added and the mixture was stirred for $15 \mathrm{~min}$. The org. layer was separated, and the aqueous phase was extracted with EtOAc. The combined org. layers were washed with saline and dried $\left(\mathrm{Na}_{2} \mathrm{SO}_{4}\right)$. After the solvent was removed, the dark residue was treated with $6 \mathrm{ml} \mathrm{Ac} \mathrm{C}_{2} \mathrm{O}$ and $1 \mathrm{ml}$ pyridine. After refluxing for $2 \mathrm{~h}$, the mixture was poured onto ice, stirred for $10 \mathrm{~min}$ and extracted with $\mathrm{CH}_{2} \mathrm{Cl}_{2}$. The org. layer was washed with water and dried $\left(\mathrm{Na}_{2} \mathrm{SO}_{4}\right)$. After evaporation, the remaining residue was chromatographed $\left(\mathrm{SiO}_{2} ; \mathrm{CH}_{2} \mathrm{Cl}_{2}\right)$ or was crystallized with $\mathrm{MeOH}$ at $3^{\circ}$. Recrystallization from EtOH or $\mathrm{MeOH}$ yielded colorless crystals.

\section{3,9-Diacetoxy-5,6-dihydro-12-methyl-indolo/2,1-a/isoquinoline (7)}

7 was synthesized from 2 b. Yield $80 \%$; m. p. $157^{\circ}$. - $\mathrm{C}_{21} \mathrm{H}_{19} \mathrm{NO}_{4}(349.4)$ Calc. C 72.2 H 5.48 Found C 71.9 H 5.59. - IR (KBr): $1760(\mathrm{CO}) \mathrm{cm}^{-1}$. ${ }^{1} \mathrm{H}-\mathrm{NMR}(90 \mathrm{MHz}): \delta(\mathrm{ppm})=2.33\left(\mathrm{~s} ; 6 \mathrm{H},-\mathrm{OCOCH}_{3}\right), 2.60(\mathrm{~s} ; 3 \mathrm{H}$, $\left.-\mathrm{CH}_{3}\right), 3.12\left(\mathrm{t} ; \mathrm{J}=7 \mathrm{~Hz}, 2 \mathrm{H},-\mathrm{CH}_{2}-\right), 4.17\left(\mathrm{t} ; \mathrm{J}=7 \mathrm{~Hz}, 2 \mathrm{H},-\mathrm{CH}_{2}-\right), 6.86$ $\left(\mathrm{dd} ; \mathrm{J}_{1 / 2}=9 / 2 \mathrm{~Hz}, 1 \mathrm{H}, \mathrm{ArH}\right), 7.11(\mathrm{~s} ; 2 \mathrm{H}, \mathrm{ArH}), 7.13\left(\mathrm{dd} ; \mathrm{J}_{1 / 2}=9 / 2 \mathrm{~Hz}, 1 \mathrm{H}\right.$, $\operatorname{ArH}), 7.6(\mathrm{~d} ; \mathrm{J}=9 \mathrm{~Hz}, 1 \mathrm{H}, \operatorname{ArH}), 7.88(\mathrm{~d} ; \mathrm{J}=9 \mathrm{~Hz}, 1 \mathrm{H}, \mathrm{ArH})$.

\section{3,9-Diacetoxy-12-formyl-5,6-dihydro-indolo/2,1-alisoquinoline (8)}

8 was synthesized from 4 b. Yield $80 \%$; m. p. $165^{\circ} .-\mathrm{C}_{21} \mathrm{H}_{17} \mathrm{NO}_{5}(363.4)$ Calc. C 69.4 H 4.71 Found C $69.6 \mathrm{H}$ 4.55. - IR (KBr): $1760\left(\mathrm{OCOCH}_{3}\right)$, $1640(\mathrm{CHO}) \mathrm{cm}^{-1}$. - ${ }^{1} \mathrm{H}-\mathrm{NMR}(90 \mathrm{MHz}): \delta(\mathrm{ppm})=2.37\left(\mathrm{~s}:{ }_{1} \mathrm{H}\right.$, $\left.-\mathrm{OCOCH}_{3}\right), 3.16\left(\mathrm{t} ; \mathrm{J}=7 \mathrm{~Hz}, 2 \mathrm{H},-\mathrm{CH}_{2}-\right), 4.2\left(\mathrm{t} ; \mathrm{J}=7 \mathrm{~Hz}, 2 \mathrm{H},-\mathrm{CH}_{2}-\right)$, 6.9-7.28 (m; 4H, ArH), $8.0(\mathrm{~d} ; \mathrm{J}=9 \mathrm{~Hz}, 1 \mathrm{H}, \operatorname{ArH}), 8.43(\mathrm{~d} ; \mathrm{J}=9 \mathrm{~Hz}, 1 \mathrm{H}$, $\mathrm{ArH}), 10.52$ (s; 1H, -CHO).

\section{2,3,9,10-Tetraacetoxy-12-formyl-5,6-dihydro-indolo/2,1-a/isoquinoline} (9)

9 was synthesized from 4 f. Yield $80 \%$; m. p. $259^{\circ}$. $-\mathrm{C}_{25} \mathrm{H}_{21} \mathrm{NO}_{9}(479.4)$ Calc. C $62.6 \mathrm{H} 4.41$ Found C $62.0 \mathrm{H}$ 4.20. - IR (KBr): $1775\left(\mathrm{OCOCH}_{3}\right)$, $1645(\mathrm{CHO}) \mathrm{cm}^{-1}$. $-{ }^{1} \mathrm{H}-\mathrm{NMR}\left(\mathrm{CDCl}_{3}\right): \delta(\mathrm{ppm})=2.37(\mathrm{~s} ; 12 \mathrm{H}$, $\left.-\mathrm{OCOCH}_{3}\right), 3.18\left(\mathrm{t} ; \mathrm{J}=7 \mathrm{~Hz}, 2 \mathrm{H},-\mathrm{CH}_{2}-\right), 4.27(\mathrm{t} ; \mathrm{J}=7 \mathrm{~Hz}, 2 \mathrm{H}$, $-\mathrm{CH}_{2}-$ ), 7.37 (s; $\left.2 \mathrm{H}, \mathrm{ArH}\right), 7.97$ (s; $\left.1 \mathrm{H}, \mathrm{ArH}\right), 8.37$ (s; $\left.1 \mathrm{H}, \mathrm{ArH}\right), 10.62$ (s; $1 \mathrm{H},-\mathrm{CHO}$ ).

\section{Biological Methods}

\section{MCF-7 Human Breast Cancer Cells ${ }^{5}$}

The MCF-7 cell line was kindly provided by Dr. M. E. Lippman, NCI, Bethesda, MD, USA. Cells were grown in improved minimal essential medium (MEM), as modified by Richter et al. ${ }^{6}$ (Biochrom, Berlin), supple 
mented with glutamine $(0.3 \mathrm{~g} / \mathrm{L})$, gentamycin $(60 \mathrm{mg} / \mathrm{L})$, and $5 \%$ newborn calf serum (NCS) (Gibco) or charcoal-treated NCS (CCS). CCS was prepared by incubation of $500 \mathrm{ml}$ of NCS with a dextran-coated charcoal pellet ${ }^{7)}$ for $4 \mathrm{~h}$ in a shaker at $4-0^{\circ}$. The procedure was repeated with a fresh pellet. After each incubation, the charcoal was removed by centrifugation. The serum was filtered through a $0.20 \mu \mathrm{m}$ filter (Sartorius) and stored at $-20^{\circ}$. Cells were grown in a humidified incubator in $5 \% \mathrm{CO}_{2}$ at $37^{\circ}$. Two weeks before start of the experiment, cells were switched from NCS to $\mathrm{CCS}$ and received two additional media changes before they were harvested with $0.05 \%$ trypsine $-0.02 \%$ EDTA in $0.15 \mathrm{M} \mathrm{NaCl}$. They were syringed gently to prevent clumping, and approximately $2 \times 10^{4}$ cells in $2 \mathrm{ml}$ were plated replicately in sixwell dishes (Costar). One day later, cells were switched to a medium containing the substances and $0.1 \%$ ethanol in which the compounds had been dissolved. The medium of control wells contained an equal volume of ethanol. At the fourth day, media were changed and substances added again. Three days later, cells were labeled with $1 \mu \mathrm{C}$ of $\left[{ }^{3} \mathrm{H}\right]$ thymidine/well for $2 \mathrm{~h}$. Cells were washed with cold PBS and harvested in PBS containing $0.02 \%$ EDTA. After centrifugation, the cell pellet was resuspended in $1 \mathrm{ml}$ of PBS and divided in two equal parts. One part was counted in a ZM Coulter counter; the other one was sonicated. After addition of $4 \mathrm{ml}$ of $10 \%$ trichloroacetic acid, the acid-insoluble fraction was collected on a $0.45 \mu \mathrm{m}$ filter (Sartorius) and counted after addition of $10 \mathrm{ml}$ of scintillation liquid (Quickszint 212, Zinsser) in a LS 1801 Beckman scintillation counter.

\section{MDA-MB 231 Human Breast Cancer Cells}

The MDA-MB 231 cell line was also provided by Dr. M. E. Lippman. Cells were grown in a McCoy 5 a medium (Boehringer Mannheim) supplemented with $10 \% \mathrm{NCS}$ and gentamycin $(40 \mu \mathrm{g} / \mathrm{ml})$. The experiments were performed as described for the MCF-7 cells with one exception: the incubation period was reduced from 6 to 2 days.

\section{P388 D Leukemia Cells}

The experimental details for the tests with $\mathrm{P} 388 \mathrm{D}_{1}$ leukemia cells have been described ${ }^{1)}$.

\section{P388 Leukemia of the Mouse}

P388 leukemia cells were generously provided by Dr. A. E. Bogden, EG \& G Mason Research Institute, Worcester, MA, USA. The tumor cells grow as ascites in the abdominal lumen of female DBA/ 2 mice (CharlesRiver-Wiga, Sulzfeld). After one week, the ascites is removed with a syringe and diluted with sterile icecold PBS to reach a cell number of $10^{7}$ cells $/ \mathrm{ml} .0 .1 \mathrm{ml}$ of this cell suspension is injected ip. into $6-10$ weeks old animals. Female $\mathrm{CDF}_{1}$-mice were used for the determination of the cytostatic activity of drugs. After the ip. injection of $10^{6}$ cells at day 0 , the animals were randomized into groups of six. $24 \mathrm{~h}$ later (day 1), and at day 5 and 9 compounds dissolved or suspended in polyethylene glycol $400 / 0.9 \%$ saline $(1: 1)$ were administered $(0.2 \mathrm{ml} /$ animal). Control animals received only the solvent.

Survival time was recorded daily. At day 1 and 5, the body weights were determined. The median survival time of treated animals was compared with that of the control group and the result is expressed as \% T/C. The decrease of body weight between day 1 and day 5 compared with control animals was used as parameter for an acute toxicity of the drugs.

\section{Estradiol Receptor Binding Assay ${ }^{8)}$}

The relative binding affinity (RBA) of the test compounds was deter mined in a competitive binding assay with $\left[{ }^{3} \mathrm{H}\right]$ estradiol. Calf uterine cytosol was incubated for $18 \mathrm{~h}$ at $4^{\circ}$ with different conc. of competitor and $5 \times$ $10^{-9} \mathrm{M}\left[{ }^{3} \mathrm{H}\right]$ estradiol. After incubation, dextran-coated charcoal was added to absorb unbound ligand $\left(90 \mathrm{~min}, 4^{\circ}\right)$ and, after centrifugation, radioactivity was determined in the supernatant using $100 \mu$ l aliquots. Six concentrations of competitor were chosen to provide values between 10 and $90 \%$ bound radioactivity. A semilogarithmic plot of bound radioactivity versus concentration was used to determine the relative binding affinity given as ratio of molar concentration of estradiol and test compound required to decrease the amount of bound radioactivity by $50 \%$, multiplied with 100.

\section{References}

1 R. Ambros, S. von Angerer, and W. Wiegrebe, Arch. Pharm. (Weinheim) 321,481 (1988).

2 D. G. O'Sullivan and P. W. Sadler, J. Chem. Soc. (London) 1959, 876.

3 E. von Angerer, personal communication.

4 R. J. Geran, N. H. Greenberg, M. M. Macdonald, A. M. Schumacher, and B. J. Abbot, Cancer Chemotherapy Rep. 3, 1 (1972).

5 E. von Angerer and J. Strohmeier, J. Med. Chem. 30, 131 (1987).

6 A. Richter, K. K. Sandford, and V. J. Evans, J. Natl. Cancer Inst. (USA) 49, 1705 (1972).

7 S. M. Scholl, K. K. Huff, and M. E. Lippman, Endocrinology (Baltimore) 113, 611 (1983).

8 J. Strohmeier and E. von Angerer, Arch. Pharm. (Weinheim) 318, 421 (1985).

[Ph 459] 


\begin{abstract}
Alle Beiträge in dieser Zeitschrift beginnen auf einer rechten Seite und nicht wie bisher im Anschluß an den vorangehenden Artikel. Es kann deshalb vorkommen, daß eine Seite teilweise oder ganz frei bleibt. Damit entsteht zwar ein etwas größerer Papierbedarf, aber die Publikationsvorbereitungen und die Herstellung der Sonderdrukke werden einfacher, so daß sich insgesamt keine zusätzlichen Kosten ergeben. Auch verlängern sich die Publikationsfristen nicht, da der Jahresumfang der Zeitschrift um die unbedruckt gebliebenen Seiten wächst.

All papers in this issue start on a new right-hand page, instead of immediately following the preceding article. Thus it may happen that part of an article's last page or even a whole (left-hand) page is blank. However, this does not result in higher costs or shorten the issue's contents. Rather, it allows faster publication, speeds up the production of reprints, and helps cutting cost increases.
\end{abstract}

